\title{
Philosophiques
}

\section{Une analyse sémantique du concept de causalité est-elle possible?}

\section{Maurice Gagnon}

Volume 2, numéro 2, octobre 1975

URI : https://id.erudit.org/iderudit/203030ar

DOI : https://doi.org/10.7202/203030ar

Aller au sommaire du numéro

Éditeur(s)

Société de philosophie du Québec

ISSN

0316-2923 (imprimé)

1492-1391 (numérique)

Découvrir la revue

Citer cet article

Gagnon, M. (1975). Une analyse sémantique du concept de causalité est-elle possible? Philosophiques, 2(2), 187-205. https://doi.org/10.7202/203030ar d'utilisation que vous pouvez consulter en ligne.

https://apropos.erudit.org/fr/usagers/politique-dutilisation/ 


\title{
UNE ANALYSE SÉMANTIQUE DU CONCEPT DE CAUSALITÉ EST-ELle POSSIBLe?
}

\author{
par Maurice Gagnon
}

Précisons tout d'abord que par analyse sémantique du concept de causalité, nous entendons ici une analyse des rapports qui existent entre les vocables cause et causalité d'une part, et d'autre part les processus !ogiques ainsi que les contenus d'expérience qui leur sont reliés. Cette analyse peut prendre la forme d'un examen des relations déductives que les propositions causales entretiennent entre elles et avec certains autres types de propositions, soit les conditionnelles subjonctives, les conditionnelles matérielles et les énoncés strictement implicatifs. Une telle étude a été faite par ie professeur Arthur W. Burks ${ }^{1}$. Nous voulons plutôt considérer ici ce que Burks appelle les "propriétés inductives $\gg^{2}$ de ces concepts, c'est-à-dire analyser les types d'expériences qui servent de fondement à la formulation ou à la confirmation d'énoncés causals, ainsi que les processus mentaux impliqués dans la formation du concept de cause.

\section{I- D'Aristote à Hume}

Entreprendre une analyse sémantique du concept de causalité, c'est d'abord se heurter à la variété infinie des verbes à résonance causale dans le langage courant: faire, provoquer, déclencher, favoriser, influencer, dominer, etc. En fait, tous les verbes actifs ont une signification causale, et tous les verbes passifs nous réfèrent à un sujet qui reçoit, subit ou se voit impo-

1. «The Logic of Causal Propositions », in Mind, LX, n. 239 (juillet 1951), pp. 363-382.

2. Ibid., pp. 363-364. 
ser l'effet d'une activité causale. Hume a estimé que le seul élément commun à cette multiplicité hétérogène et bigarrée est l'idée de production d'un résultat quelconque par un agent. Effectivement, cette idée de production est présente dans la définition aristotélicienne de cause efficiente : «...ce dont vient le premier commencement du mouvement et du repos; par exemple, l'auteur d'une décision est cause, le père est cause de l'enfant, et, en général, l'agent est cause de ce qui est fait, ce qui produit le changement de ce qui est changé ${ }^{3}$.

L'idée de production semble également présente dans le traitement que donne la scolastique du concept de cause. Témoin les définitions suivantes : «'Ce qui fait l'être', ce dont dépend l'existant et, par suite seulement, ce qui le rend intelligible ${ }^{4}$; «la cause désignait pour Aristote ce par quoi un tel existe, et aussi ce par quoi il est rendu intelligible, compréhensible ${ }^{5}$; «ce dont une chose dépend quant à l'existence ${ }^{8}$, «ce par quoi quelque chose est ${ }^{7}$.

Enfin, cette idée de production transcende les querelles épistémologiques entre le réalisme et le criticisme kantien, puisque Kant, dans la première édition de la Critique de la Raison Pure, intitule ainsi la section sur la seconde analogie de l'expérience: «Deuxième analogie. Principe de la production. Tout ce qui arrive (commence d'être) suppose quelque chose à quoi il succède, d'après une règle ${ }^{8} ;$ la seconde édition remplace ce dernier texte par le suivant: "Principe de la succession dans le temps suivant la loi de la causalité. Tous les changements se produisent suivant la loi de la liaison de la cause et de l'effet. ${ }^{9}$ Dans sa preuve de la seconde analogie ${ }^{10}$, Kant distingue deux types de succession: la succession subjective et relative de nos

3. Pbysique, traduction Carteron, Les Belles Lettres, 1952, 194b 29-33; voir aussi idem, 195a 22-24 et Métaphysique, traduction Tricot, Vrin, 1962, A, 3, 938a 30-31 et 2, 1013a 29-33.

4. MUNIER, A., Manuel de Philosopbie, Desclée et Cie, 1956, tome I, p. 108.

5. AUBERT, J.-M., Pbilosopbie de la nature, Beauchesne, 1965, p. 46.

6. JOLIVET, R., Traité de Pbilosopbie, tome III, p. 328.

7. Id., ibid., p. 331.

8. Critique de la Raison Pure, trad. Tremesaygues et Pacaud, P.U.F., 1965, p. 182.

9. Ibid., note 1.

10. Ibid., pp. 182-195. 
perceptions d'un même phénomène qui ne nous apparaît pas en train de changer, et la succession objective qui se situe dans le phénomène lui-même, et non pas seulement dans les appréhensions variées que nous en avons. Cette succession objective comporte un ordre nécessaire et infaillible, asymétrique. Comme il s'agit de phénomènes et non pas de choses en soi, la seule explication possible de cet ordre est qu'il a sa source en nous, dans une règle synthétique et a priori ; cette règle dit qu'un état donné succède infailliblement à un état antérieur déterminé, mais pas l'inverse. Cette règle n'est pas tirée de l'expérience par induction, mais on la trouve plutôt dans une analyse réflexive de la manière dont nous coordonnons nos propres perceptions. Par elle, nous sommes renvoyés d'un état présent en tant qu'arrivé à un état antérieur qui est corrélatif du premier, bien que peut-être encore inconnu quant à sa nature. Ce corrélatif détermine la conséquence de l'état arrivé et le rattache nécessairement à lui-même dans la succession temporelle.

À l'objection possible que l'usage ordinaire du principe de causalité admet la simultanéité de la cause et de l'effet, alors que le traitement qu'en fait la philosophie critique semble le restreindre à un certain type de succession temporelle, Kant répond premièrement que la cause et l'effet sont simultanés au moment où l'effet commence à se produire, mais que très souvent la cause ne peut produire son effet en même temps; d'autre part, même quand l'effet et la cause sont simultanés, le rapport qui les lie est toujours déterminable dans le temps : ainsi, par exemple, l'existence et le mouvement du poids que je dépose sur un coussin mou précèdent l'écrasement du coussin. La succession objective est donc l'unique critère empirique permettant de rattacher l'effet aux causes qui le précèdent. Kant ajoute que le concept de cause nous renvoie à celui d'action, lequel nous renvoie à celui de force, qui nous mène lui-même à celui de substance. La substance constitue le sujet ultime et permanent de ce qui change et ce sont les changements d'état de la substance qui requièrent une cause, et non la permanence d'un état: un corps en état de mouvement uniforme n'a pas besoin de cause pour demeurer indéfiniment en cet état, mais il faut une cause pour modifier sa vitesse ou sa trajectoire. Se demandant enfin comment se font ces changements' d'état, Kant affirme que la réponse à cette ques- 
tion n'est pas a priori et relève exclusivement de recherches empiriques sur les forces réelles qui amènent les changements d'état, ou, en d'autres termes, sur certains phénomènes déterminés qui se succèdent toujours.

L'usage des termes comme «production》, «nécessité 》, «action » et «force » révèle que la notion kantienne de causalité ne diffère pas substantiellement quant à son contenu sémantique des notions mentionnées plus haut. Ce qui est nouveau chez Kant, c'est le statut ontologique des entités reliées par une relation causale : la causalité réaliste d'Aristote et des scolastiques appartient à des objets en soi et son asymétrie est un rapport nécessaire fondé sur les natures réciproques de la cause et de l'effet; la causalité kantienne existe entre des phénomènes et son asymétrie dépend d'un a priori cognitif.

Hume a cependant rejeté cette idée de production, car il l'a jugée trop vague et trop obscure pour être utile à un esprit féru de clarté et de précision. Il en est de même pour toutes les autres notions associées à l'idée de production, soit celles de pouvoir, force, efficacité, nécessité, etc. ${ }^{11}$. Hume propose donc une nouvelle définition de la cause, où cause et effet doivent satisfaire aux conditions suivantes ${ }^{12}$ :

1. Être constamment conjoints,

2. être contigus dans l'espace et le temps,

3. la cause doit être antérieure,

4. aux causes d'un même genre correspondent toujours des effets d'un même genre,

5. les variations de la cause et de l'effet sont proportionnelles (ce que Stuart Mill appellera plus tard variation concomitante),

6. la cause qui se présente parfois sans son effet n'en est pas la cause totale.

11. A Treatise of Human Nature, Everyman's Library, Dutton, 1964, pp. 80, 93, 153-170.

12. Ibid., pp. 170-172. 
Du même souffle, Hume déclare pétitions de principe certaines tentatives visant à démontrer que «tout ce qui arrive doit avoir une cause », et il soutient que la nécessité ne nous est pas donnée dans l'expérience et ne saurait donc être justifiée par une induction. Elle n'est fondée, selon lui, que sur une association d'idées, ce qui la rend tout à fait illusoire.

Il est clair que Hume abandonne la notion courante et aristotélicienne de cause pour en proposer une autre: aucune des clauses de la définition humienne ne semble absolument indispensable à la notion courante, mais l'item de production, que Hume rejette, l'est. La nouvelle définition renonce à dire en quoi consiste la relation causale, elle se borne à énumérer les caractères distinctifs des objets reliés par cette relation. Cette énumération n'est pas arbitraire et a priori : elle se fonde sur l'expérience et conserve plusieurs aspects de la notion courante, mais en éliminant ce que cette dernière comporte d'obscur et de méta-expérimental. Hume a voulu formuler une notion de cause qui soit acceptable en fonction de la mécanique de Newton, toute nouvelle à son époque : sa conception de la causalité s'applique particulièrement bien à l'étude des vitesses et des trajectoires en relation avec les forces d'accélération et de résistance appliquées par contact. Mais il se trouve déjà dans la physique newtonienne un élément rebelle à la causalité humienne : c'est la force d'attraction gravitationnelle agissant à distance. Les clauses 1,2 et 3 de la définition mentionnée plus haut ne s'y appliquent pas, et l'usage du terme «force» révèle que Newton fait appel à la notion courante de cause. Il est vrai qu'il avoue ne pas savoir en quoi consiste cette force d'attraction, et ajoute que cela n'est pas nécessaire: il suffit de pouvoir établir un rapport entre d'une part ses effets, à savoir les trajectoires et les vitesses effectives, et d'autre part les masses et les distances.

\section{II-Hume dépassé}

Cette dernière attitude de Newton annonce une tendance qui milite en faveur de l'abandon du concept de cause pour celui de fonction. Cette tendance s'affirme définitivement avec Faraday qui, vers le milieu du 19e siècle, rejette l'idée d'action à distance dans l'explication des phénomènes électro-magnétiques, pour ne parler que de champ. En 1894, Hertz écrit ses Principes de 
Mécanique, proposant une reconstruction de la mécanique où la force disparaît comme notion fondamentale pour être remplacée par celle de liaison entre les masses. Il est généralement admis par ceux qui s'intéressent aujourd'hui à l'épistémologie de la science que Hume avait raison, mais qu'il n'est pas allé assez loin, du moins en fonction du contexte scientifique actuel. Nous allons examiner et critiquer dans les lignes qui suivent quelques tentatives pour donner à la notion de relation causale un statut propre et un sens précis, et nous essaierons ensuite de faire le bilan de ces tentatives.

Dans un article publié en $1965^{13}$, J.-L. Mackie propose la définition suivante: une cause est une "partie non suffisante mais nécessaire d'une condition qui est elle-même non nécessaire mais suffisante pour que le résultat se produise 》 ( an insufficient but necessary part of a condition which is itself unnecessary but sufficient for the result»; en raccourci, «INUS condition »). La condition non nécessaire mais suffisante est l'une parmi toutes les conjonctions de facteurs dont l'ensemble est suffisant pour amener le résultat, et la totalité de ces conditions constitue une série disjonctive possiblement infinie. Par exemple, l'incendie d'un édifice peut être provoqué par l'un ou l'autre des ensembles de facteurs suivants :

- court-circuit, présence d'oxygène, présence de matières inflammables, absence d'extincteur, etc.;

- action d'un incendiaire, présence d'oxygène, présence de matières inflammables, distraction du veilleur de nuit, etc. ;

- etc...

Le tout peut être formalisé en une disjonction possiblement infinie de conjonctions:

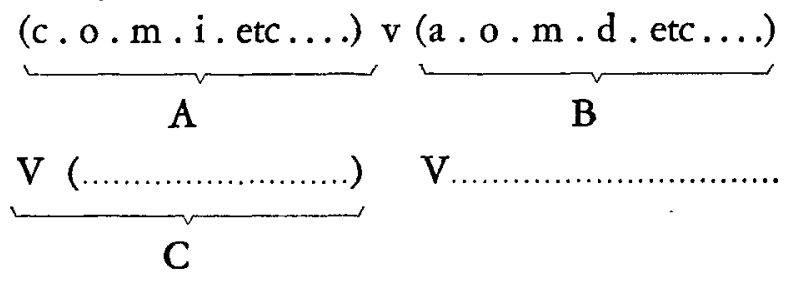

13. "Causes and Conditions», dans American Pbilosopbical Quarterly, vol. 5, n. 4 , pp. $245-264$. 
où $\mathrm{A}, \mathrm{B}, \mathrm{C}$, etc., sont les conditions non nécessaires mais suffisantes mentionnées plus haut. Mackie les appelle aussi «conditions suffisantes minimales ». Chacun de leurs éléments est nécessaire pour que le tout constitue une condition suffisante, et chacun est une "INUS condition »: cause partielle choisie parmi les autres en fonction du point de vue particulier sous lequel on examine la situation. Ainsi pour un inspecteur municipal la cause de l'incendie sera le court-circuit, tandis que pour l'enquêteur de la compagnie d'assurance ce sera plutôt la négligence du propriétaire qui n'a pas placé un extincteur chimique au bon endroit, etc.

Pour rendre plus précise la désignation de la cause, Mackie propose la notion de champ causal. On impose ainsi des limites à la série disjonctive exposée plus haut, ce qui a pour effet de situer l' «INUS condition 》 à l'intérieur d'un ensemble déterminé. On dira, par exemple, que tel virus cause la grippe chez les êtres humains, et que tel état physique cause la grippe de certaines gens, c'est-à-dire les empêche de résister efficacement, comme le font d'autres personnes, au virus de la grippe. Dans le premier cas, le champ causal englobe les êtres humains en général ; dans le second, il comprend les êtres humains attaqués par le virus de la grippe. Le champ causal peut être restreint jusqu’à ne comprendre que les événements biochimiques qui ont eu lieu pendant une période déterminée de la vie de Pierre : il permettra alors la formulation d'un énoncé causal concernant la grippe que Pierre a contractée à cette époque. Le champ causal est une région plus large à l'intérieur de laquelle il y a une sous-région qui se distingue par une caractéristique dont on cherche la cause: c'est ici que l' "INUS condition » entre en scène ${ }^{14}$. Et Mackie montre sans difficultés que son analyse est valable pour les énoncés causals singuliers ${ }^{15}$ comme pour les énoncés généraux ${ }^{16}$, et que même la dépendance fonctionnelle établie par la méthode des variations concomitantes peut être traduite en termes d' "INUS condition $\gg$ relativement à un champ causal déterminé ${ }^{17}$.

14. Ibid., pp. 248-249.

15. Ibid., pp. $245-252$.

16. Ibid., pp. $252-253$.

17. Ibid., pp. 260-261. 
L'élaboration de Mackie est un raffinement de la conception humienne. L'item «condition nécessaire » maintient la clause de conjonction constante, car c'est par cette dernière seulement qu'on peut s'assurer de la nécessité d'une condition. Mais cette conjonction n'a besoin d'être constante qu'à l'intérieur de certaines limites. $\bar{A}$ l'intérieur de la condition minimale suffisante dont elle fait partie, l' «INUS condition» doit faire l'objet d'une conjonction constante avec l'effet: les courts-circuits ne sont pas tous accompagnés d'un incendie, mais le sont ceux dont l'arrivée coïncide avec la présence de matières inflammables, la présence d'oxygène, l'absence d'extincteur ou de surveillant. La nécessité dont on parle ici est différente de celle que rejette Hume. Elle signifie seulement qu'on n'a jamais observé de fait l'événement $B$ sans l'événement $A$, et non pas que l'arrivée de $A$ entraîne infailliblement celle de B, ou qu'il est impossible d'avoir B sans A. Quant à l'item de «condition suffisante», il s'applique à chaque condition suffisante minimale, et il est évident qu'aucune d'entre elles n'est en conjonction constante avec l'effet, sauf le cas fort peu probable où le champ causal ne contiendrait qu'une seule de ces conditions minimales suffisantes. Il suffit qu'à l'intérieur du champ causal chaque condition minimale soit plus ou moins fréquemment conjointe à l'effet. Sur cette base, on peut faire des statistiques établissant la fréquence respective des différentes causes suffisantes d'un même effet, et des calculs de probabilité concernant ces diverses causes. Il en est de même pour une «INUS condition» qui serait membre de plusieurs conditions suffisantes minimales du même effet. Si une «INUS condition » est membre de toutes les conditions suffisantes minimales possibles pour un même effet à l'intérieur d'un champ causal déterminé, alors on pourra parler de sa conjonction constante avec l'effet dans ce champ causal.

Mackie abandonne la clause de contiguïté spatio-temporelle, de même que celle de la priotité temporelle de la cause. Il maintient que la relation causale est asymétrique, mais cette asymétrie est intemporelle : «A cause $B$ » veut simplement dire qu'on n'obtient pas B sans A relativement à un certain champ causal, l'inverse n'étant pas forcément vrai ; de plus, B ne précède jamais A, mais les deux peuvent être soit simultanés, soit immédiatement successifs, soit séparés par un délai. Cette asymétrie intemporelle 
n'est ni posée a priori, ni démontrée, ni expliquée, mais simplement constatée: la friction d'une allumette sur une surface appropriée cause son ignition, mais l'inverse n'a jamais été observé ; et quand un incendie cause un court-circuit, ce n'est pas la même relation causale que lorsqu'un court-circuit cause un incendie. L'asymétrie nous renvoie à des descriptions de processus physiques, psychologiques ou sociaux, ces descriptions étant conditionnées par les limites de nos connaissances actuelles. Ainsi modifiée, et isolée des autres ingrédients humiens, la conjonction constante devient une fonction asymétrique entre des variables déterminées. L'expression «INUS condition » nous rappelle que le premier terme de cette fonction est habituellement un élément d'un tout fort complexe.

Mario Bunge ${ }^{18}$ propose une notion de cause qu'il juge acceptable pour le physicien contemporain, et qu'il définit de la façon suivante :

Une relation peut être dite causale si et seulement si :

1) les relata sont des propriétés ou des événements appartenant à deux systèmes différents, le déterminant et le déterminé ;

2) les relata sont régulièrement conjoints, c'est-à-dire simplement ou stochastiquement conjoints ;

3) il doit y avoir un délai, positif ou nul, entre la cause et l'effet. En d'autres termes, l'effet n'est jamais antérieur à la cause ;

4) la réaction du déterminé sur le déterminant est plus faible que l'action du déterminant sur le déterminé ;

5) les variations du déterminé sont nulles ou négligeables. Autrement dit, il n'y a pas d'effets spontanés;

6) les causes et les effets doivent être en relation biunivoque (i.e. l'application des inputs sur les outputs doit être bijective) ${ }^{19}$.

18. "Conjonction, succession, détermination, causalité », in Les théories de la causalité, Paris, P.U.F., 1971, pp. 112-132.

19. Ibid., pp. 129-130. 
À première vue, ce texte nous rappelle la notion aristotélicienne de cause efficiente: la cause produit ou fait exister l'effet, la qualité de l'effet est proportionnelle à celle de la cause, et la relation causale possède une asymétrie non temporelle. Mais les clauses 4) et 5) de la définition de Bunge ne garantissent nullement que le déterminant produit le déterminé. Ce n'est donc pas tout à fait la notion courante et aristotélicienne, bien que la définition de Bunge s'en rapproche davantage que celle de Hume : chez Bunge comme chez Aristote, l'asymétrie de la relation causale fait de la cause une variable indépendante à qui il est simplement interdit d'être postérieure à son effet, variable dépendante. Il faut ajouter que Bunge admet le principe de causalité, non à titre de principe métaphysique, mais à titre de postulat méthodologique indispensable pour fonder la science physique, ce qui explique la clause 5) de sa définition. La nécessité est donc introduite dans la relation causale sous la forme d'un postulat, mais cette nécessité n'implique pas nécessairement le concept de production mentionné plus haut. De plus, la clause 2) ci-haut remplace fort heureusement la notion de conjonction constante par celle de conjonction régulière, qui peut être simple (constante) ou stochastique, c'est-à-dire que la probabilité conjointe des deux événements considérés n’est pas nulle ${ }^{20}$. Ceci apporte des précisions utiles qui ne sont pas clairement formulées par Mackie, bien qu'impliquées par sa conception générale, comme nous l'avons montré plus haut. La clause 6) de la conception de Bunge marque un désaccord avec Mackie en ce qu'elle élimine les cas où une mêtne cause accompagne alternativement des effets différents, et le cas où plusieurs causes accompagnent alternativement le même effet ${ }^{21}$. La notion de cause chez Mackie a une dénotation plus large cue chez Bunge, et se rapproche davantage de celle du langage courant. Mais par-delà cette divergence, Bunge fait lui aussi de la causalité une fonction asymétrique entre des variables ou des systèmes de variables.

Carnap a tenté de justifier un usage scientifique de la relation causale en la ramenant à la dérivation logique ${ }^{22}$. Dire que

20. Ibid., pp. 115-121.

21. Ibid., p. 130.

22. Pbilosopbical Foundations of Pbysics, Basic Books, 1966, chap. 19, pp. 187. 195. 
l'événement A cause l'événement B signifie que d'un ensemble $\mathrm{L}$ de lois (l'existence même de cet ensemble étant tout à fait problématique si les lois en question ne sont pas toutes actuellement connues) jumelé à une description de $A$, on peut dériver logiquement un énoncé décrivant $\mathrm{B}$. La causalité est donc synonyme de «dérivabilité potentielle» (《potential predictability» selon les termes de Carnap : le mot «prediction» a ici un sens plus large que la prévision d'un fait futur, mais signifie la déduction logique d'un événement passé ou futur à partir de certaines prémisses).

Soulignons tout d'abord que cette notion de «dérivabilité potentielle », que Carnap lui-même qualifie de métaphorique, est plutôt élastique et donne lieu à une infinité d'énoncés causals problématiques dont la vérité n'est pas actuellement vérifiable et ne le sera peut-être jamais. En outre, quel doit être le contenu d'une loi du genre de celles dont parle Carnap? Il nous paraît à la fois suffisant et nécessaire qu'elle affirme la conjonction régulière, constante ou stochastique, et asymétrique de $\mathrm{A}$ et de $\mathrm{B}$ à l'intérieur d'un champ déterminé de phénomènes. Nous ne voyons pas comment il pourrait en être autrement, peu importe que la «loi » en question soit dérivée de l'expérience ou qu'elle soit posée a priori comme structure théorétique: la seule différence est que dans le second cas une vérification expérimentale s'impose. Seule une loi de ce type peut, conjointement avec une description de $A$, servir de prémisse à la déduction logique d'une description de B. Nous avons ainsi tous les éléments voulus pour définir la cause dans les termes de Mackie, et nous sommes ainsi ramenés à l'idée de fonction asymétrique.

Herbert Simon a tenté de définir la causalité en termes de rapports d'inclusion entre des ensembles de lois déterminant des propositions atomiques ${ }^{23}$. II serait trop long ici d'entrer dans tous les détails de cette conception dont nous ne donnons que les grandes lignes.

23. Models of Man, New York, Wiley, 1957, chap. 3: On the Definition of the Causal Relation. Cet article fut d'abord publié dans Journal of Philo. sopby, vol. 49, juillet 1952. 
Un ensemble $\mathrm{L}$ de lois détermine une proposition atomique a si, et seulement si les descriptions $\mathrm{D}_{1}, \mathrm{D}_{2} \ldots \ldots \ldots \ldots . . . \mathrm{D}_{\mathrm{n}}$, où figurent a ou $-a$, sont les seules telles que ( $L \& D_{1} \& D_{2}$ \& ............. \& $\left.D_{n}\right)$ n'est pas un énoncé contradictoire. En d'autres termes, seules les descriptions contenant a ou -a sont compatibles avec L. Supposons maintenant que nous avons les ensembles de lois $L$ et $L^{\prime}$ déterminant respectivement les propositions atomiques a et a'. Si L. et L' sont disjoints, alors a et a' sont indépendantes; si $L$ et $L^{\prime}$ sont identiques, alors a et a' sont dans un rapport de dépendance réciproque ; si L' est un sous-ensemble propre de $L$, alors a' précède causalement a relativement à l'ensemble (L,L'). Simon arrive à montrer de cette manière que «il pleut» précède causalement «Jean porte son imperméable»; il en est de même successivement pour « les pluies sont plus abondantes que d'habitude cette année », " il y a une très forte récolte de blé cette année » et «le prix du blé est très bas cette année ».

Évidemment, les hiérarchies causales ainsi découvertes ne correspondent pas toujours à celles du langage courant; et à l'inverse, on ne réussit pas toujours à trouver des ensembles de lois dont les rapports d'inclusion correspondent aux hiérarchies causales du langage courant: on ne décide pas arbitrairement des lois de la nature. Il se peut aussi que plusieurs ensembles disjoints de lois déterminent la même proposition atomique. C'est pourquoi l'invariance de la relation causale n'est pas garantie: il peut arriver que a précède causalement $b$ par rapport à l'ensemble de lois $K$, et que $b$ précède causalement a par rapport à l'ensemble de lois K'. Simon arrive à sauver cette invariance par une solution ad boc fondée sur une distinction fort artificielle entre deux types de propositions atomiques empiriques, les unes décrivant des observations, les autres des conditions.

Dans un article posiérieur ${ }^{24}$, Simon évite cette difficulté en utilisant des systèmes indépendants, consistants et «selfcontained» (c'est-à-dire contenant autant d'équations que de variables distinctes) d'équations linéaires non homogènes. Nous

24. Causal Ordering and Identifiability, dans Studies in Econometric Methods, édité par William C. Hood et Tjalling C. Koopmans, New York, Wiley, 1953, chap. III. Réimprimé comme le précédent article dans Models of Man, chap. I, pp. 10-36. 
ne pouvons que renvoyer le lecteur à ce texte technique, dense et fort difficile à résumer. Mais le premier paragraphe de la conclusion de Simon vaut d'être cité :

In this chapter we have defined a concept of causality that corresponds to the intuitive use of that term in scientific discussion. Causality is an asymmetrical relation among certain variables, or subsets of variables, in a self-contained structure. There is no necessary connection between the asymmetry of this relation and asymmetry in time, although an analysis of the causal structure of dynamical systems in econometrics and physics will slow that lagged relations can be interpreted as causal relations.

Sur la double base de la psychologie génétique et de l'histoire des sciences, Jean Piaget propose un examen diachronique de la notion de cause ${ }^{25}$, projetant ainsi une certaine lumière sur la critique humienne et sur les rapports entre l'usage pré-scientifique de la notion de cause et d'autres usages plus sophistiqués. Issue chez le jeune enfant de l'expérience tactilo-kinesthésique, plus précisément de l'expérience de manipuler, pousser ou lancer des objets, la causalité serait d'abord perçue sous la forme de l'efficacité de l'action propre sur les choses, le moi et les choses constituant à l'origine un tout indifférencié. Au fur et à mesure que son expérience du monde se raffine, se précise et se diversifie, le sujet modifie progressivement cette notion primitive de causalité. Elle est peu à peu décentrée du moi et attribuée à tous les objets sans exception, ce qui explique partiellement l'animisme enfantin. Le phénoménisme initial, où le monde était perçu comme une série de tableaux successifs dont on pourrait dire: «esse est percipi», est remplacé par un univers d'objets donnés de permanence et d'individualité, et le sujet devient conscient de la nécessité des connexions mécaniques dans les séquences causales, tout au moins dans celles où sont en cause des objets fami-

25. Voir Jean PIAGET, Introduction à l'épistémologie génétique, tome II, Paris, P.U.F., 1950; La construction du réel cbez l'enfant, 4e édition, Neuchatel, Delachaux et Niestlé, 1967 ; Six études de psychologie, Collection Médiations, Paris, Gonthier, 1964; La psycbologie de l'intelligence, Collection U2, Paris, Armand Colin, 1967. Voir aussi M. Bunge, F. Halbwacks, Th. S. Kuhn, J. Piaget et L. Rosenfeld, Les théories de la causalité, Paris, P.U.F., 1971; J. Piaget et R. Garcia, Les explications causales, Paris, P.U.F., 1971. 
liers et manipulables. C'est dire que l'évolution du concept de cause prend déjà une direction humienne, tout en conservant encore le facteur d'efficacité (et donc aussi de production et de nécessité) comme un de ses éléments indispensables. Le rejet de ces derniers éléments viendra éventuellement pour ceux qui recevront une éducation scientifique et se consacreront à des analyses de plus en plus abstraites.

S'inspirant de ces considérations, de même que des recherches de Michotte sur la perception de la causalité ${ }^{26}$, Piaget affirme que l'usage d'un langage causal est lié pour la plupart des gens à la perception d'un contact entre les objets, suivi de changements dans leurs vitesses et leurs trajectoires, ces changements étant relatifs à la masse de chacun. Ceci est « l'équivalent perceptif d'une sorte de calcul des vitesses ${ }^{27}$. Cette conception de la causalité n'est pas forcément anthropomorphique, car le contact est dissociable des sensations d'effort et de poussée. D'ailleurs, la contiguité spatiale est l'un des caractères de la causalité explicitement reconnu par Hume, bien que ce dernier ait été farouchement opposé à toute forme d'anthropomorphisme. Mais lorsqu'on ne peut repérer de contact entre les choses, comme c'est le cas dans les théories physiques du champ et dans l'examen des rapports des phénomènes psychologiques et sociaux entre eux, le terme causalité devient synonyme de fonction asymétrique. Si l'on tient absolument à parler de la relation causale comme d'une relation non mathématique, la notion de causalité devient alors vicariante, c'est-à-dire qu'elle exprime ce qui constitue, dans le monde en soi, le parallèle problématique et invérifiable de certaines relations mathématiques mises en oeuvre par le langage scientifique.

Vu que la conjonction régulière asymétrique de variables justifie l'énoncé de lois à partir desquelles des faits peuvent être prédits avec succès, la notion de causalité devient aussi synonyme d'explication, spécialement lorsque les lois ou théories en question contiennent des termes purement théoriques, c'est-à-dire dont le référent n'est pas empiriquement constaté. La perspective de

26. MICHOTTE, A., La perception de la causalité, 2e édition, Louvain, Publications Universitaires de Louvain, 1954.

27. Introduction à l'épistémologze génétique, tome II, p. 275 
Piaget rejoint partiellement ici celle de Carnap, la différence étant que Piaget ne parle pas d'une simple dérivation possible, mais d'une dérivation effective, confirmée sans être asservie par l'expérience. Il est possible que cette dérivation logique ait pour parallèle une causalité physique, mais l'existence de cette dernière est d'autant plus problématique que l'existence même des référents des termes purement théoriques est également problématique, et qu'on ne possède aucun critère objectif de découpage des phénomènes en événements individuels.

\section{III-Conclusion}

Les quatre tentatives de définition de la causalité que nous avons examinées convergent sur un point: la réduction de la notion de causalité à des complexes de relations mathématiques va de pair avec la formalisation progressive des sciences. Si l'on continue à y parler de causalité, c'est en un sens presqu'entièrement nouveau, car il semble n'avoir qu'un seul point commun avec le sens préscientifique: l'asymétrie. Parler de causalité au sens de production efficace n'a de sens que dans un contexte concret où des êtres humains sont directement impliqués comme producteurs. Ici, le concept de relation causale n'est probablement pas issu de la seule expérience tactilokinesthésique, mais aussi de l'expérience d'être façonné, influencé par les idées et les coutumes de son milieu social, et d'exercer soi-même une semblable influence sur d'autres. Le terme causalité revêt alors un sens fort vague, comme l'a bien vu Hume, parce qu'il exprime une infinie variété d'expériences dont le contenu varie selon les contextes, selon le type de chose à produire et le mode de production utilisé. Cette variété, il faut le souligner fortement, défie toute analyse sémantique précise : il y a autant de sens précis du terme cause qu'il y a de combinaisons possibles entre contexte, objet à produire et mode de production. Il est sans aucun doute possible de formuler des définitions de ces causalités particulières, mais chacune de ces définitions devra être opérationnelle, c'est-à-dire décrire les opérations effectuées (et les instruments utilisés quand c'est le cas) pour obtenir un résultat déterminé.

Dès qu'on quitte le niveau du vécu pour élaborer des schèmes logico-mathématiques très précis mais ambigus en ce sens 
qu'ils peuvent être appliqués à des situations concrètes aux contenus extrêmement variés, la notion de causalité productrice n'a plus de place. Même en supposant que les situations en question contiennent des relations causales, on ne peut en tirer un caractère commun qui soit assez simple et assez précis pour figurer dans les schèmes formels. Si l'on parle encore de causalité, c'est uniquement comme synonyme de conjonction régulière asymétrique. De telles conjonctions sont constatées expérimentalement, mais hors les cas de l'activité humaine fabricatrice et peut-être de la mécanique classique, on ne peut dire que l'une des variables concernées produit l'autre.

Une précision nous paraît ici nécessaire. Lorsque nous affirmons que la notion scientifique de causalité se ramène à certaines relations ou complexes de relations mathématiques, nous parlons $\mathrm{du}$ volet purement théorique de la science. Il en va autrement dans les arts et les sciences appliquées. Un traité de physique ou de biologie n'a pas à parler de cause productrice; mais la situation est tout autre dans la pratique du génie ou de la médecine, où on essaie de produire, empêcher ou modifier certains phénomènes. Ici, l'agent humain est personnellement impliqué : il agit sur les phénomènes soit directement, soit par l'intermédiaire d'instruments spécifiques qu'il doit manipuler selon une méthode et un ordre bien déterminés. Stephen Toulmin dit fort justement sur cette question :

The theories which are produced to explain optical phenomena, for instance, do not specifically tell us how to bring about this or that optical effect - how to produce a shadow a hundred feet deep, or how to create a mirage. Rather they provide us with a picture of the sorts of phenomena to be expected in any given circumstances, which can then be used in any number of ways. The study of the causes of this or that event is, therefore, always an application of physics. It is not of direct importance to the physicist, and can at best suggest to him something which may turn out to be of theoretical importance. In the case of theories, as of maps, there will be an indefinite number of applications to be made, say, in engineering. But the way in which the theory is formulated will not show that it is to be applied in this or that particular kind of way, for the production or prevention of this or that particular kind of development. Problems of application and questions about causes arise with 
reference to particular contexts, but physical theories are formulated in a manner indifferent to particular contexts: it is when we come to apply theories that we read off from them the causes of this and that, but there is no call for the term 'cause' to figure within the theories themselves ${ }^{28}$.

On peut rapprocher ce texte des affirmations de Piaget sur la causalité perçue d'abord comme efficacité dans le contexte de l'expérience tactilo-kinesthésique : les deux témoignent du caractère fondamentalement pragmatique de la notion de cause productrice. Elle est essentiellement liée à l'efficacité de certaines actions émanant d'un agent humain. Dans le cas d'une activité couronnée de succès (c'est-à-dire que le résultat attendu se produit), cet agent et les divers instruments dont il se sert peuvent être appelés chacun à sa façon cause productrice. Si l'activité en question échoue, on recherchera alors la cause de cet échec: erreur de l'agent humain, faillite d'un instrument, usage d'une mauvaise théorie ou d'instruments inappropriés, etc. Cette notion de cause productrice peut, comme nous l'avons dit plus haut, être transposée sans anthropomorphisme dans la mécanique classique, où la résistance des corps et leurs chocs réciproques jouent un rôle important. Mais il ne semble pas possible d'aller au-delà.

Nous nous retrouvons finalement avec deux notions de causalité : l'une préscientifique et pragmatique, l'autre scientifique, les deux n'ayant en commun que l'asymétrie. La première est vague dans la mesure où elle s'applique à des situations concrètes fort variées, d'où le fait qu'elle est synonyme de production. La description de telle production concrète et déterminée peut être relativement précise si elle se fonde sur nos connaissances scientifiques actuelles. Mais cette analyse descriptive n'est jamais sûre d'épuiser tous les intermédiaires possibles entre la cause principale et l'effet, elle peut être raffinée à l'infini. En d'autres termes, on ne peut jamais être sûr que la liste de tous les facteurs nécessaires à la production de tel objet déterminé est complète et définitive. Ici, il y a autant d'analyses sémantiques

28. The Pbilosopby of Science. An Introduction, New York, Harper \& Row, 1960 , p. 122. Sur cette question, toute la section intitulée «Causes are the concern of the applied sciences», dans le même volume, pp. 119-124, est à lire. 
précises qu'il y a de productions individuelles, chacune de ces analyses étant un processus toujours inachevé, perfectible à l'infini. Mais il n'y a pas d'analyse sémantique générale et précise qui soit valable pour tous les cas possibles. Prenons, en guise d'exemple, les deux propositions causales suivantes: «Jean a fabriqué une table » et "Paul a effrayé Jeanne en portant un masque grotesque ». L'analyse de la signification de ces deux énoncés mènerait à des descriptions fort différentes : la première se ferait en termes de la nature des instruments utilisés, de la résistance des matériaux, et peut-être même des propriétés moléculaires de ces derniers; la seconde description parlerait d'abord de réactions physiologiques complexes consécutives à un certain stimulus lumineux, puis de réactions psychologiques probablement encore plus complexes dont nous ne connaissons encore que relativement peu de choses. Chaque description serait fort incomplète, mais on pourrait appeler «production» chacun des événements ainsi décrits.

La seconde notion de causalité est purement formelle, elle fait abstraction de tout contexte particulier. «Cause » veut dire alors : "variable indépendante qui est en relation de conjonction régulière asymétrique avec une autre variable dite dépendante $»$; et «effet»: «variable dépendante qui est en relation de conjonction régulière asymétrique avec une autre variable, dite indépendante ». Le contenu des variables ainsi reliées n'a aucune importance et c'est pourquoi nous avons une notion de causalité claire et univoque.

Nous nous trouvons ici devant un exemple de ce que Bachelard appelle la coupure épistémologique entre la connaissance du sens commun et la connaissance scientifique. Suite à une analyse plus circonstanciée et plus raffinée des phénomènes, le scientifique en arrive à des synthèses totalement différentes de celles du sens commun, qui sont basées sur une analyse trop limitée. Si le scientifique, dans la formulation de sa synthèse, utilise le même vocabulaire que le sens commun, alors les mêmes mots y ont une signification différente parce qu'utilisés dans des contextes différents; et ces diverses significations n'ont que très peu, voire même pas du tout, de rapports entre eux. Bachelard s'est d'ailleurs moqué du «choquisme » de ceux qui prétendaient 
transposer au niveau des particules subatomiques les lois causales de la mécanique classique ${ }^{29}$.

Bachelard a fait le «profil épistémologique » de deux idées fondamentales en physique: masse et énergie ${ }^{30}$. Dans les deux cas, il y a fractionnement en cinq notions différentes, correspondant à cinq étapes du progrès de la pensée : la notion du sens commun y est suivie de celle de l'empirisme positiviste, puis viennent ensuite successivement celles de la physique newtonnienne, de la physique relativiste et de la physique de Dirac. On obtient ainsi une série de notions de plus en plus abstraites, chacune assignant des limites aux précédentes, chacune ayant son champ d'application particulier. Il serait intéressant de tenter aussi le profil épistémologique de la notion de causalité. On y retrouverait certainement, dans un ordre d'abstraction croissante, la causalité-production du sens commun, et la relation de conjonction asymétrique décrite plus haut. La notion humienne (et peut-être aussi la notion kantienne) constituerait entre les deux un intermédiaire valable, correspondant à une certaine étape du progrès de la pensée rationnelle.

Université de Sherbrooke

29. BACHELARD, G., L'activité rationaliste de la pbysique contemporaine, P.U.F., 1951, pp. 83-87.

30. BACHELARD, G., La pbilosopbie du non, P.U.F., 1962, pp. 41-51. 